\section{FRI0671 ULTRA-HIGH FIELD MRI AND BIOMECHANICAL INVESTIGATION OF VERTEBRAL BONE MICROARCHITECTURE}

S. Guis ${ }^{1}$, D. Guenoun ${ }^{2}$, A. Fouré ${ }^{3}$, M. Pithioux ${ }^{4}$, T. Le Corroller ${ }^{2}$, J.P. Mattei ${ }^{1}$, V. Pauly ${ }^{5}$, M. Bernard ${ }^{3}$, P. Chabrand ${ }^{4}$, P. Champsaur ${ }^{2}$, D. Bendahan ${ }^{3}$.

${ }^{1}$ Rheumatology $1 ;{ }^{2}$ Radiology, Aix Marseille Université, AP-HM; ${ }^{3}$ CRMBM, UMR 7339; ${ }^{4}$ Ism UMR 7287, CNRS; ${ }^{5}$ Statistic department, AP-HM, Marseille Cedex 9, France

Background: Fracture risk prediction in patient relies chiefly on bone mineral density (BMD) measurement. However, the decreased bone strength characteristic of osteoporosis is dependent not only on BMD, but also on other factors, most notably bone microarchitecture.

Objectives: The purpose of this study was to investigate bone microarchitecture variables of cadaveric vertebrae using ultra-high field MRI (7 Tesla).

Methods: Twenty four vertebrae (L2, L3, L4) from eight cadavers were studied using 7 Tesla MRI. Their Bone Mineral Density (BMD) were investigated using dual energy X Ray absorptiometry. Then, all specimens underwent mechanical compression tests to failure and the failure load (in Newton) and constraint (in Mpa) were measured. Bone Volume Fraction (BV/TV), Trabecular Thickness (Tb.Th), and Trabecular Spacing (Tb.Sp) were measured in MR images using a Digital topological analysis (Bone J). Measurements were performed by two observators in order to characterize the inter-rater reliability. Statistical analyses were performed using SPSS. Correlations between variables were analyzed using Spearman correlations and Stepwise regression. A p value of 0.05 was considered as significant.

Results: The inter-rater reliability for bone microarchitecture parameters quantification was good. Tb.Th and Tb.Sp measured using high-field MRI were $0.52 \pm 0.18$ and $0.48 \pm 0.10$ respectively while the BV/TV fraction was $0.52 \pm 0.13$. The mean BMD was $0.86 \pm 0.20 \mathrm{~g} / \mathrm{cm}^{2}$. The failure load and the constraint measured during the compression tests were $2600 \pm 1267 \mathrm{~N}$ and $1.57 \pm 0.81 \mathrm{Mpa}$ respectively. Interestingly, the variables measured during the mechanical tests were significantly

The failure load and constraint measured during the compression tests were significantly correlated with the BMD. Regarding the bone indices quantified using high-field MRI, a significant linear relationship was observed between the trabecular spacing and the $\operatorname{BMD}\left(R^{2}=0.23, p=0.01\right.$ and the constraint values to failure $\left(R^{2}=0.18, p=0.04\right)$. A stepwise regression with backward elimination demonstrated that combining BV/TV and BMD improved the relationship with the constraints from an adjusted $R^{2}=0.384$ for $\mathrm{BMD}$ alone to an adjusted $R^{2}=0.41$ for $\mathrm{BMD}+\mathrm{BV} / \mathrm{TV}$.

Conclusions: In the present study, we demonstrated for the first time that the variables characterizing the vertebral bone microarchitecture quantified using ultra-high field MRI were significantly correlated with biomechanical parameters. In addition, we illustrated that the vertebral bone strength was better described by a variable combining BMD and trabecular bone spacing.

Disclosure of Interest: None declared

DOI: 10.1136/annrheumdis-2017-eular.2034

\section{FRI0672 ASSESSMENT OF THE NAIL BED IN PSORIATIC ARTHRITIS (PSA) BY ULTRASOUND (US) AND MRI}

S. Paramalingam ${ }^{1}$, A. Taylor ${ }^{2}$, H. Keen ${ }^{2,3} \cdot{ }^{1}$ Rheumatology, Sir Charles Gairdner Hospital; ${ }^{2}$ Rheumatology, Fiona Stanley Hospital; ${ }^{3}$ School of Medicine, University of Western Australia, Perth, Australia

Background: PsA can be difficult to distinguish from osteoarthritis (OA) and the treatments vary greatly. Nail involvement can occur in about $15-50 \%$ patients with psoriasis ${ }^{1}$, which may aid differentiation from OA. If US can reliably detect nail bed changes in early disease then it might be helpful in differentiating PsA from OA Objectives: This study aims to investigate the nail bed changes seen in patients with PsA and OA using US and MRI, and to determine the impact of nail bed changes on quality of life (QOL).

Methods: Patients who fulfilled the CASPAR PsA or 1990 ACR OA classification criteria were recruited. At baseline, clinical assessment included patient and physician's visual analogue scale (VAS), tender and swollen joints, patient's Leeds Enthesitis Index and quality of life specific to nail psoriasis (NPQ10). Nail abnormalities on the dominant hand such as onycholysis, pitting, nail bed hyperkeratosis and nail bed crumbling were documented. Using US, each nail (1-5) was scored dichotomously for pitting/irregularity, loss of normal trilaminar appearance of the nail. Each nail was scored semi quantitatively for power doppler (PD) signal (0-3) in the nail bed, nail matrix and dermis, and assigned a total PD score. All patients had an MRI of the dominant 2 nd to 5 th finger

Results: 14 patients were recruited; demographics, clinical and US detected nail changes are documented in Table 1. Clinical nail changes were not seen in the OA group, but were relatively common in the PsA group. In the PsA group, $54 \%$ of nails had US detected structural abnormalities; $12 \%$ pitting and $52 \%$ loss of trilaminar layer. Only $20 \%$ of OA nails had US detected abnormalities. There was a strong relationship between the presence of clinical nail change and US structural changes (chi square 10.769 df $1 \mathrm{p}=0.001$ ) but not $\mathrm{PD}$ signal score. There was no relationship between clinical or US detected nail scores and NPQ10, patient or physician VAS, swollen joint count or Leeds Enthesitis Index. MRI analysis is pending.
Table 1

\begin{tabular}{lccc}
\hline & Total $(\mathrm{n}=14)$ & PsA $(\mathrm{n}=10)$ & OA $(\mathrm{n}=4)$ \\
\hline Age (median (IQR)) & $56.5(52.2-63.2)$ & $55.0(48.0-63.2)$ & $61.0(55.2-73.5)$ \\
Patient VAS (median (IQR)) & $29.0(12.5-54.8$ & $39.5(19.8-54.8)$ & $11.0(3.8-54.2)$ \\
Physician VAS (median (IQR)) & $35.3(20.5-57.5)$ & $42.5(24.5-57.5)$ & $24.0(4.8-53.8)$ \\
NPQ10 (median (IQR)) & $6.5(1.8-10.0)$ & $7.0(5.2-10.2)$ & $1.5(0.2-5.8)$ \\
Swollen joint count (median (IQR)) & $2.0(0.0-4.2)$ & $1.5(0.0-5.2)$ & $2.5(0.5-5.8)$ \\
Leeds Enthesitis index (median (IQR)) & $1.0(0.0-2.0)$ & $1.0(0.0-2.0)$ & $0.5(0.0-1.8)$ \\
Percentage of nails with clinical & & & \\
$\quad$ abnormalities & $27.7 \%$ & $40.0 \%$ & $0.0 \%$ \\
$\begin{array}{l}\text { Percentage of nails with US structural } \\
\quad \text { abnormalities }\end{array}$ & $44.3 \%$ & $54.0 \%$ & $20.0 \%$ \\
Percentage of nails with PD signal & $97.1 \%$ & $96.0 \%$ & $95.0 \%$ \\
\hline
\end{tabular}

Conclusions: In this small prospective study, structural abnormalities detected by US appear to correlate well against clinical structural findings, but PD signal does not. No relationship was found between US findings and other clinical parameters. US structural abnormalities may be a better differentiator of PSA and OA than PD signal. MRI validation of these results is pending.

References:

[1] Gisondi P, Idolazzi L, Girolomoni G. Ultrasonography reveals nail thickening in patients with chronic plaque psoriasis. Arch Dermatol Res 304:727-732,2012. Acknowledgements: This study was funded by a grant from UCB Australia Pty Ltd

Disclosure of Interest: None declared

DOI: 10.1136/annrheumdis-2017-eular.5614

\section{FRI0673 CLINICAL REMISSION IN TOCILIZUMAB-USING RHEUMATOID ARTHRITIS PATIENTS CAN BE OVERESTIMATED: A CROSS SECTIONAL STUDY USING ULTRASOUND SONOGRAPHY}

S. Nakabo ${ }^{1}$, Y. Tsuji ${ }^{2}$, M. Inagaki ${ }^{2}$, H. Tsuji ${ }^{1}$, T. Nakajima $^{1}$, M. Hashimoto ${ }^{3}$, M. Furu ${ }^{3,4}$, M. Tanaka ${ }^{3}, \mathrm{H}$. Ito ${ }^{4}$, T. Fujii ${ }^{1,5}$, Y. Fujii ${ }^{2}$, T. Mimori ${ }^{1}$. ${ }^{1}$ Department of Rheumatology and Clinical Immunology; ${ }^{2}$ Human Health Sciences Clinical Physiology and Ultrasound Labo; ${ }^{3}$ Department of the Control for Rheumatic Diseases; ${ }^{4}$ Department of Orthopaedic Surgery, Graduate School of Medicine, Kyoto University, Kyoto; ${ }^{5}$ Department of Clinical Immunology and Rheumatology, Wakayama Medical University, Wakayama, Japan

Background: Several clinical remission (CR) criteria of rheumatoid arthritis (RA) contain acute-phase reactants [C-reactive protein (CRP), and erythrocyte sedimentation rate $(E S R)]$ as their components. However, it is known that they can be underscored by the usage of tocilizumab (TCZ). This may cause overestimation of CR. On the other hand, ultrasound sonography (US) provides objective information independent of acute-phase reactants and assessment by physician and patient, and can detect synovitis in RA patients in CR, which is called sonographic residual synovitis (SRS).

Objectives: To assess whether CR of TCZ-using RA patients are overestimated, by using US.

Methods: We recruited 402 RA patients. Bilateral 2-5 MCP, wrist, ankle, and 2-5MTP joints were scanned by using the Aplio500 (TOSHIBA) with a $12 \mathrm{MHz}$ transducer. Power Doppler (PD) images were obtained by Superb Micro-vascular Imaging (SMI). Gray scale (GS) and PD images were scored using a 0-3 semiquantitative scale. Clinical information was obtained from the Kyoto University Rheumatoid Arthritis Management Alliance (KURAMA) database, which is based on the assessments by physicians who were blind to the US results. The patients were divided into 4 groups based on their treatment: tumor necrosis factor alpha inhibitors (TNFi), TCZ, abatacept (ABT), and non-biologic (non-Bio) users Two patients treated by tofacitinib were excluded because the number was too small to analyze. The Boolean (BL)-based, Simplified Disease Activity Index (SDAI)-based, Clinical Disease Activity Index (CDAl)-based, and Disease Activity Score (DAS)28-ESR-based CR criteria were assessed using SRS.

Results: A total of 400 RA patients were analyzed. The number of patients in each treatment group is shown in the Table. When the BL-based, SDAI-based, and DAS28-ESR-based CR criteria were used, SRS in TCZ group was significantly stronger than the other groups (Fig 1-3). On the other hand, when CDAl-based $\mathrm{CR}$ criteria was used, the difference was not significant among 4 groups (Fig

\begin{tabular}{lccccc}
\hline & TNFi & TCZ & ABT & Non-Bio & $P$ \\
& $\mathrm{~N}=104$ & $\mathrm{~N}=35$ & $\mathrm{~N}=42$ & $\mathrm{~N}=219$ & \\
\hline Age, years & $62.8 \pm 13.4$ & $60.0 \pm 11.6$ & $64.8 \pm 11.1$ & $63.7 \pm 13.0$ & 0.28 \\
Sex (M/F) & $14 / 90$ & $8 / 27$ & $2 / 40$ & $48 / 171$ & 0.03 \\
Disease duration, years & $12.3 \pm 11.6$ & $12.0 \pm 11.3$ & $11.5 \pm 9.5$ & $13.1 \pm 11.2$ & 0.75 \\
Steinbrocker Stage (I/I//II//V) & $28 / 26 / 17 / 33$ & $6 / 9 / 7 / 13$ & $10 / 11 / 6 / 15$ & $57 / 48 / 49 / 65$ & 0.73 \\
PhGA, mm & $9.9 \pm 11.9$ & $9.1 \pm 14.2$ & $11.6 \pm 15.5$ & $9.2 \pm 10.2$ & 0.94 \\
PtGA, mm & $26.0 \pm 22.5$ & $27.6 \pm 23.2$ & $26.9 \pm 26.2$ & $25.4 \pm 24.6$ & 0.73 \\
CRP, mg/dL & $0.4 \pm 0.8$ & $0.2 \pm 0.6$ & $0.6 \pm 1.0$ & $0.5 \pm 0.9$ & $<0.01$ \\
ESR, mm/hr & $22.8 \pm 18.6$ & $11.2 \pm 11.5$ & $26.7 \pm 19.2$ & $22.8 \pm 17.6$ & $<0.01$ \\
Tender joint count & $0.8 \pm 1.3$ & $0.7 \pm 1.5$ & $1.4 \pm 2.9$ & $1.0 \pm 1.6$ & 0.27 \\
Swollen joint count & $0.8 \pm 1.6$ & $0.7 \pm 1.1$ & $1.3 \pm 2.6$ & $0.9 \pm 1.8$ & 0.94 \\
Total GS score & $11.1 \pm 5.7$ & $14.2 \pm 8.1$ & $12.5 \pm 5.9$ & $13.9 \pm 7.4$ & $<0.01$ \\
Total PD score & $2.6 \pm 3.9$ & $4.6 \pm 5.1$ & $3.0 \pm 4.0$ & $3.5 \pm 4.9$ & 0.04 \\
MTX, mg/week & $6.0 \pm 4.2$ & $4.2 \pm 4.6$ & $3.0 \pm 3.6$ & $5.4 \pm 4.1$ & $<0.01$ \\
Prednisolone dose, mg/day & $0.9 \pm 2.0$ & $1.3 \pm 2.3$ & $1.8 \pm 3.4$ & $1.1 \pm 2.3$ & 0.19 \\
\hline
\end{tabular}

Mean \pm SD. 
4). In TCZ group, CRP and ESR were significantly lower than the other groups, although other clinical indicators were comparable (Table).

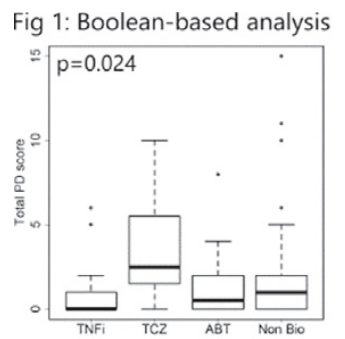

Fig 3: DAS28-ESR-based analysis

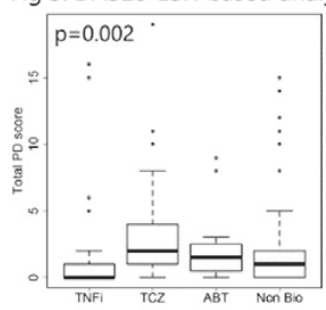

Fig 2: SDAI-based analysis

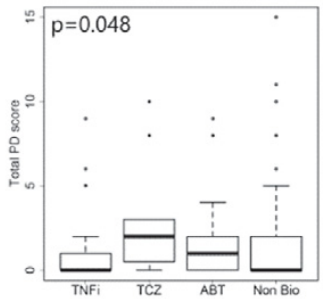

Fig 4: CDAl-based analysis

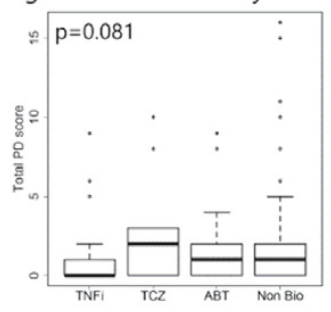

Kruskal-Wallis rank sum test
Conclusions: US revealed that CR in TCZ-using can be overestimated by BL-based, SDAl-based, and DAS28-ESR-based CR criteria. For TCZ users, CDAl-based CR criteria is more reliable than the other criteria.

Disclosure of Interest: None declared

DOI: 10.1136/annrheumdis-2017-eular.1705

\section{FRI0674 USING HIGHER IMAGE RESOLUTION OF MAGNETIC RESONANCE IMAGING OF THE CERVICAL SPINE IDENTIFIES MORE INFLAMMATORY AND STRUCTURAL LESIONS IN PATIENTS WITH AXIAL SPONDYLOARTHRITIS}

S. Krabbe ${ }^{1}$, M. Østergaard ${ }^{1}$, J. Møller ${ }^{2}$, I.J. Sørensen ${ }^{1}$, B. Jensen ${ }^{1}$, O.R. Madsen 1 , S.J. Pedersen ${ }^{1} .{ }^{1}$ Center for Rheumatology and Spine Diseases, Rigshospitalet, Copenhagen; ${ }^{2}$ Department of Radiology, Herlev Hospital, Herlev, Denmark

Background: The vertebrae of the cervical spine are rather small and it may be difficult to assess if small areas with signal intensity changes represent the bones, joints or entheses, or derive from the surrounding blood vessels.

Objectives: To investigate if image resolution affects the assessment of inflammatory and structural lesions of the cervical spine.

Methods: Forty-nine patients with axial spondyloarthritis according to the ASAS criteria started anti-TNF treatment and had "standard" resolution (std-res) and "high" resolution (high-res) MRI sequences of the cervical spine performed at baseline and after 48 weeks. 3 patients had follow-up scan already after 6-24 weeks due to study exclusion.

Std-res: STIR sequence: Voxel size $5.0 \mathrm{~mm}^{3}$ (slice thickness 4.0, spatial resolution 1x1.25); T1W sequence: voxel size $4.5 \mathrm{~mm}^{3}$ (slice thickness 4.0, spatial resolution $0.9 \times 1.25)$.

High-res: STIR sequence: Voxel size $3.1 \mathrm{~mm}^{3}$ (slice thickness 3.5 , spatial resolution 0.8x1.11); T1W sequence: voxel size $1.4 \mathrm{~mm}^{3}$ (slice thickness 3.0 , spatial resolution $0.6 \times 0.76$ ).

Images were assessed in known chronology by an experienced axSpA MRI reader (SJP) blinded to clinical data. High-res and std-res were read in random order. MRI lesions of inflammation, fat and new bone formation were defined according to the Canada-Denmark working group [1,2]. Erosions were not assessed.

Results: Inflammatory lesions: In 9 of 43 patients (21\%), inflammatory lesions were detected in the cervical spine at baseline at std-res, while this was detected in 14 of 43 patients (33\%) at high-res. Using high-res, as compared to std-res, 6 patients were reclassified from negative to positive for inflammation, 1 patient was reclassified from positive to negative, and $8 / 28$ patients remained classified as positive/negative, $\mathrm{p}=0.13$ by Exact McNemar test. The mean inflammation score was significantly higher at high-res compared to std-res (1.7 (SD 4.5) vs. 0.8 (SD 2.7), $p=0.04$ by paired t-test).

Fat lesions: 11 of 43 patients $(26 \%)$ had fat lesions in the cervical spine at baseline using std-res, while 10 of 43 patients $(23 \%)$ had this using high-res. The mean fat score was significantly higher at high-res compared with std-res (1.6 (SD 3.5) vs. 0.8 (SD 1.8), $\mathrm{p}=0.02$ by paired t-test).

Bone spurs/ankylosis: 11 of 43 patients $(26 \%)$ had bone spurs/ankylosis of the cervical spine at baseline at std-res, while 10 of 43 patients $(23 \%)$ using high-res. The mean new bone formation score was significantly higher at high-res compared with std-res (2.7 (SD 6.1) vs. 1.4 (SD 3.5), $\mathrm{p}=0.01$ by paired t-test).

Responsiveness: Standardized response mean for inflammation score at std-res was 0.15 , and at high-res 0.14 . Structural lesions remained largely unchanged in all patients.
Conclusions: More patients were classified as having inflammatory lesions in the cervical spine when using high-res MRI, compared to std-res. Likewise, mean scores of inflammatory lesions, fatty lesions and new bone formation were significantly higher compared with std-res. Further studies are needed to investigate the clinical significance of these findings as well as the frequency of these minor lesions in healthy controls.

ClinicalTrials.gov: NCT01029847.

References:

[1] Lambert RGW, et al. J Rheumatol 2009;S84:3-17.

[2] Østergaard M, et al. J Rheumatol 2009;S84:18-34.

Disclosure of Interest: None declared

DOI: 10.1136/annrheumdis-2017-eular.3200

\section{FRI0675 OBTAINING SYNOVIAL BIOPSIES FROM THE WRIST IN PATIENTS WITH NEWLY DIAGNOSED UNTREATED AND LONGSTANDING RHEUMATOID ARTHRITIS FOLLOWED BY INTRAMUSCULAR GLUCOCORTICOID AND METHOTREXATE INITIATION IS SAFE AND THE GLUCOCORTICOID TREATMENT SIGNIFICANTLY REDUCES DISEASE ACTIVITY}

S.A. Just $^{1}$, C. Nielsen ${ }^{2}$, E.K. Hejbø| ${ }^{3}$, H.D. Schrøder ${ }^{3}$, I.M.J. Hansen ${ }^{4}$, T. Barington ${ }^{2}$, H.M. Lindegaard ${ }^{1}$. ${ }^{1}$ Rheumatology; ${ }^{2}$ Clinical Immunology; ${ }^{3}$ Clinical Pathology, Odense University Hospital, Odense; ${ }^{4}$ Medicine, Svendborg Hospital, Odense University Hospital, Svendborg, Denmark

Background: The minimal invasive ultrasound-guided synovial biopsy (USG-SB) method has been shown to be safe and tolerable. The method has accelerated the research field of using synovial biopsies focusing on early diagnosis, disease stratification, biomarker studies and in the future optimal treatment selection for the individual patient. Here synovial biopsies obtained from patients with early arthritis before therapy initiation are essential. A major issue in newly diagnosed RA patients but also in RA patients with longstanding active RA, is to combine an effective fast working treatment with safely obtaining synovial tissue without delaying treatment initiation. In the EULAR early arthritis recommendations, prompt treatment initiation is recommended by combining glucocorticoid bridge therapy with disease-modifying antirheumatic drugs (DMARD). It is therefore essential that accepting synovial biopsy, does not delay start of fast remissioninducing treatment. Especially if synovial biopsies by the USG-SB method in the future shall be used systematically for detailed disease stratification and personalized treatment decisions.

Objectives: Safety of using intramuscular glucocorticoid injection (IGI) immediately after the USG-SB procedure in patients with newly diagnosed untreated RA or longstanding active RA, and the effect of IGI on disease activity after 4 weeks. Methods: Wrist synovial biopsies were taken) at inclusion and after 6 months from 22 patients with newly diagnosed, untreated RA and 15 with longstanding RA (>5 years). After biopsies patients were offered an IGI of $2 \mathrm{ml}$ of methylprednisolone acetate (Depo Medrol) $40 \mathrm{mg} / \mathrm{ml}$. Early RA patients were also started on methotrexate. Disease activity scores in 28 joints (DAS28) were recorded at day of biopsy and again after 4 weeks. Safety data were obtained after 5 days (telephone), 2 weeks (questionnaire) and at first clinical evaluation (4 weeks) after biopsy. Patient-reported outcomes (PRO) with pain, swelling and stiffness of biopsied joint were obtained at day of biopsy and after two weeks.

Results: At present time, all patients have undergone first biopsy and $18 / 37$ second biopsy. At the EULAR congress complete data will be presented. $68 \%$ of all patients accepted IGI after first biopsy currently 39\% after second procedure. Patients accepting IGI after first biopsy did not have higher DAS-28 (early RA group $(p=0.15)$, longstanding $R A(p=0.06))$. Time to first follow-up was not significantly different for patients accepting IGI (early RA group $(p=0.17$ ), longstanding RA $(p=0.05))$. Two weeks after biopsy, PRO was not significantly different when comparing IGI vs non-IGI treated. For all patients, DAS-28 was significantly reduced in the group receiving IGI at first clinical evaluation after synovial biopsy ( $p=0.004$, without IGI $\triangle$ DAS28: -0.5 , with IGI $\triangle$ DAS28: -1.7 ). Conclusions: Start of treatment with IGI combined with DMARD after obtaining synovial biopsies by the USG-SB procedure from patients with early untreated $\mathrm{RA}$ and longstanding RA is safe, and reduces disease activity more than without IGI.

Disclosure of Interest: None declared

DOI: 10.1136/annrheumdis-2017-eular.2491

\section{FRI0676 A PROPOSAL FOR A SIMPLE ULTRASOUND METHOD FOR} THE DIAGNOSIS OF EARLY RHEUMATOID ARTHRITIS

T. Serban ${ }^{1,2}$, I. Satulu $^{3}$, O. Vutcanu ${ }^{1}$, M. Milicescu ${ }^{1,2}$, C.M. Mihai ${ }^{1,2}$ M. Bojinca ${ }^{1,2} .{ }^{1}$ Internal Medicine and Rheumatology, "Dr. I. Cantacuzino" Clinical Hospital; ${ }^{2}$ Internal Medicine and Rheumatology, "Carol Davila" University of Medicine and Pharmacy, Bucharest, Romania; ${ }^{3}$ Rheumatology, Kalmar County Hospital, Kalmar, Sweden

Background: At this point, the classification criteria for rheumatoid arthritis (RA) are well known and generally applied in clinical practice. (1) Ultrasound (US assessment can help in distinguishing the patients with early RA (ERA) within the patients with early inflammatory arthritis (EIA).

Objectives: The aim of this study was to develop an US method for the diagnosis 